\title{
Infinitely many small energy solutions for fractional coupled Schrödinger system with critical growth
}

\author{
Peiluan Li ${ }^{a, *}$, Yuan Yuan ${ }^{b}$, Yuanxian Hui ${ }^{c}$ \\ a School of Mathematics and Statistics, Henan University of Science and Technology, Luoyang 471023, China. \\ ${ }^{b}$ Department of Mathematics and Statistics, Memorial University of Newfoundland, St. John's NL A1B 3X7, Canada. \\ ${ }^{c}$ School of Mathematics and Statistics, Puer University, Puer 665000, China.
}

Communicated by D. Baleanu

\begin{abstract}
In this paper, we investigate the small energy solutions for a coupled fractional Schrödinger system with critical growth. The existence criteria of infinitely many small energy solutions are established without Ambrosetti-Rabinowitz (A-R) condition by variant fountain theorem. Our main results are completely new and complement the previously known studies. C 2017 All rights reserved.
\end{abstract}

Keywords: Coupled fractional Schrödinger system, small energy solutions, variational methods, critical growth, variant fountain theorem.

2010 MSC: 26A33, 34B37, 34K10.

\section{Introduction}

In the present paper, we consider the following coupled Schrödinger system involving fractional pLaplacian

$$
\begin{cases}(-\Delta)^{\alpha} u(x)+V_{1}(x) u=F_{u}(x, u, v)+|u|^{2_{\alpha}^{*}-2} u, & x \in \mathbb{R}^{N}, \\ (-\Delta)^{\alpha} v(x)+V_{2}(x) v=F_{v}(x, u, v)+|v|^{2_{\alpha}^{*}-2} v, & x \in \mathbb{R}^{N},\end{cases}
$$

where $0<\alpha<1,2 \alpha<N, 2_{\alpha}^{*}=\frac{2 N}{N-2 \alpha}, V_{1}, V_{2} \in C\left(\mathbb{R}^{N}, \mathbb{R}\right), F \in C\left(\mathbb{R}^{N} \times \mathbb{R}^{2}, \mathbb{R}\right),(-\Delta)^{\alpha}$ is the so-called fractional Laplacian operator of order $\alpha$ and can be either defined pointwise for $x \in \mathbb{R}^{N}$ by

$$
(-\Delta)^{\alpha} u(x):=\text { P.V. } \int_{R^{N}} \frac{u(x)-u(y)}{|x-y|^{N+2 \alpha}} d y,
$$

where P.V. stands for the principal value, or be characterized as by $(-\Delta)^{\alpha} \mathfrak{u}(x)=\mathcal{F}^{-1}\left(|\xi|^{2 \alpha} \mathcal{F} \mathfrak{u}\right), \mathcal{F}$ denotes the usual Fourier transform in $R^{N}$, for the detail, see [12]. The nonlinearities $F_{u}(x, u, v)$ and $F_{v}(x, u, v)$ denote the partial derivatives of the function $F(x, u, v)$, which satisfies some assumptions that are necessary for the main results and will be stated in the sequence.

\footnotetext{
*Corresponding author

Email addresses: lpllpl_lpl@163.com (Peiluan Li), yyuan@mun.ca (Yuan Yuan), 949014655@qq.com (Yuanxian Hui)
} 
Over the past decades, with the aid of different methods, the existence and multiplicity of the nontrivial solutions for the integer order Schrödinger equation have been extensively investigated. The fractional $(-\Delta)^{\alpha}$ reduces to the standard Laplacian operator $-\Delta$ as $\alpha \rightarrow 1$, which was proved in [12]. Hence the results in this paper are also valid for $\alpha \rightarrow 1$.

Fractional calculus provides a powerful tool for the description of hereditary properties of various materials and memory processes. Fractional differential equations have played an important role in many fields such as engineering, science, electrical circuits, diffusion, and applied mathematics, see [17, 19, 26, $31]$ and so on. For more details about the linear and nonlinear fractional partial differential equations and their use to the description of a series of phenomena in applied sciences, see [1-3, 8, 18, 25, 35, 37].

Recently, a great attention has been focused on the study of problems involving the fractional Laplacian. $(-\Delta)^{\alpha}$ may be viewed as the infinitesimal generators of a Levy stable diffusion processes (see [5]). This operator arises in the description of various phenomena in the applied sciences, such as phase transitions, materials science, conservation laws, minimal surfaces, water waves, optimization, plasma physics and so on, see [12] and references therein. The fractional Schrödinger equation is a fundamental equation in the field of fractional quantum mechanics. It was discovered by Laskin [20, 21] as a result of extending the Feynman path integral. In recent few years, may researchers have investigated the existence and multiplicity of (critical) fractional Schrödinger equations, see for instance $[6,9,13,15,24,27,29,30,32,33,36,38]$. In some work, the nonlinearity satisfies the AmbrosettiRabinowitz (A-R) condition, i.e., there exists $\theta>2$ such that $0<\theta \mathrm{F}(x, t)<\operatorname{tf}(x, t)$. But in some other papers, the authors obtained their results without A-R condition.

On the other hand, the coupled quasi-linear Schrödinger system involving Laplacian appears in several branches of physics. It can accurately describe the multiplicate chemical reaction catalyzed by the catalyst grains under constant or variant temperature, and a correspondence of the stable station of the dynamical system determined by the reactiondiffusion system. It also arises in non-Newtonian fluids, flow through porous media, nonlinear elasticity, glaciology, quasi-regular and quasi-conformal mappings. When the potentials $a(x)$ and $b(x)$ are coercive in $\mathbb{R}^{N}$, Costa [11] studied a class of coupled semilinear Schrödinger system:

$$
\begin{cases}-\Delta u+a(x) u=F_{u}(x, u, v), & x \in \mathbb{R}^{N}, \\ -\Delta v+b(x) v=F_{v}(x, u, v), & x \in \mathbb{R}^{N},\end{cases}
$$

where $F \in C^{1}\left(\mathbb{R}^{N} \times \mathbb{R}^{2}, \mathbb{R}\right)$. By applying the generalized mountain pass lemma, the author established the existence of one nontrivial solution of (1.2). From then on, the coupled quasi-linear Schrödinger system had attracted more and more attention, see $[4,10,14,22,23,28]$ and the references therein. But for the coupled quasi-linear Schrödinger system involving fractional Laplacian, there are very few work. In [16], the authors considered the following coupled quasi-linear Schrödinger system involving fractional Laplacian:

$$
\begin{cases}(-\Delta)^{s} u+u=\left(|u|^{2 p}+b|u|^{p-1}|v|^{p+1}\right) u, & x \in \mathbb{R}^{N}, \\ (-\Delta)^{s} v+w^{2 s} v=\left(|v|^{2 p}+b|v|^{p-1}|u|^{p+1}\right) v, & x \in \mathbb{R}^{N},\end{cases}
$$

where

$$
0<s<1, \quad 2<2 p+2<2_{s}^{*}:=\left\{\begin{array}{cl}
+\infty & \text { if } N \leqslant 2 s \\
\frac{2 N}{N-2 s} & \text { if } N>2 s
\end{array}\right.
$$

By use of the s-harmonic extension technique, they established the existence of a nontrivial least energy solution of the system via variational methods. Especially, in the autonomous case, i.e., $b(x)=b$, $a$ positive least energy solution with both nontrivial components was obtained.

Motivated by the works above, in the present article, we investigate the system (1.1). The existence criteria of infinitely many energy solutions are established without A-R condition by fountain theorem. Obviously, the form of (1.1) is more general than that of (1.2)-(1.3). Hence our results can be viewed as an extension to the results in $[4,10,11,14,16,22,23,28]$.

To state our main results, we make the following assumptions. 
$\left(G_{1}\right) V_{i}$ is bounded from below by a positive constant, for $i=1,2$;

$\left(G_{2}\right)$ there exists a positive constant $R$ such that $\lim _{|y| \rightarrow \infty} \operatorname{meas}\left(\left\{x \in B_{R}(y): V_{i}(x) \leqslant c\right\}\right)=0$ for any $c>0$ and $i=1,2$, where $B_{R}(y)=\left\{x \in \mathbb{R}^{N}:|x-y|<R\right\} ;$

$\left(\mathrm{H}_{0}\right) \mathrm{F} \in \mathrm{C}^{1}\left(\mathbb{R}^{\mathrm{N}} \times \mathbb{R}^{2}, \mathbb{R}\right)$ such that $\mathrm{F}(\mathrm{x}, \mathrm{u}, v)>0$ if $(\mathrm{u}, v) \neq(0,0), \mathrm{F}(\mathrm{x}, 0,0)=0$;

$\left(\mathrm{H}_{1}\right)$ there exist two positive constants $r \in[1,2)$ and $a>0$ such that

$$
\left|\mathrm{F}_{z}(x, z)\right| \leqslant \mathrm{a}\left(1+|z|^{\mathrm{r}-1}\right), \quad \forall(x, z) \in \mathbb{R}^{\mathrm{N}} \times \mathbb{R}^{2}, \quad z=(u, v),
$$

where $F_{z}(x, z)=\left(F_{u}(x, z), F_{v}(x, z)\right),|z|=|(u, v)|=\sqrt{u^{2}+v^{2}} ;$

$\left(H_{2}\right) \lim _{|(u, v)| \rightarrow 0} \frac{F(t, u, v)}{|(u, v)|^{2}}=0$ uniformly in $x \in \mathbb{R}^{N}$, where $|(u, v)|=\sqrt{u^{2}+v^{2}}$;

$\left(\mathrm{H}_{3}\right)$ there exist two constants $\sigma \in[1, r)$ and $b>0$ such that $\lim _{|(u, v)| \rightarrow \infty} \frac{F(x, u, v)}{|(u, v)|^{\sigma}} \geqslant b$ uniformly for $x \in R^{N}$.

It is easy to see that $\left(G_{2}\right)$ is weaker that the coercivity assumption that $V(x) \rightarrow \infty$, as $|x| \rightarrow \infty$, which was first given in [7] to overcome the lack of compactness.

The main result of this paper is the following.

Theorem 1.1. Assume that $\left(\mathrm{G}_{1}\right)-\left(\mathrm{G}_{2}\right),\left(\mathrm{H}_{0}\right)-\left(\mathrm{H}_{3}\right)$ hold, and $\mathrm{F}(\mathrm{x},-\mathrm{u},-v)=\mathrm{F}(\mathrm{x}, \mathrm{u}, \mathrm{v})$ for all $(\mathrm{x}, \mathrm{u}, v) \in \mathbb{R}^{\mathrm{N}} \times \mathbb{R}^{2}$, then (1.1) has infinitely many small energy solutions $\left(u^{(k)}, v^{(k)}\right) \in X \backslash\{0\}$, for every $k \in N$, in the sense that

$$
\frac{\left\|\left(\mathrm{u}^{(\mathrm{k})}, v^{(\mathrm{k})}\right)\right\|_{\mathrm{X}}^{2}}{2}-\left[\int_{\mathrm{R}^{\mathrm{N}}} \mathrm{F}\left(\mathrm{x}, \mathrm{u}^{(\mathrm{k})}, v^{(\mathrm{k})}\right) \mathrm{d} x+\frac{1}{2_{\alpha}^{*}} \int_{\mathrm{R}^{\mathrm{N}}}\left(\left|\mathrm{u}^{(\mathrm{k})}\right|^{2_{\alpha}^{*}}+\left|v^{(\mathrm{k})}\right|^{2_{\alpha}^{*}}\right) \mathrm{d} x\right] \rightarrow 0^{-}, \text {as } \mathrm{k} \rightarrow \infty .
$$

The rest of this paper is organized as follows. In Section 2, some lemmas which are essential to prove our main results are stated. In Section 3, we give the main results.

\section{Preliminaries}

First, we review some related lemmas, which will be used further in this paper.

For any fixed $t \in R^{N}$ and $1<q \leqslant \infty$, we define the following norms:

$$
\|u\|_{\infty}=\max _{t \in R^{N}}|u(t)|, \quad\|u\|_{q}=\|u\|_{L^{q}\left(R^{N}\right)}=\left(\int_{R^{N}}|u(s)|^{q} d x\right)^{\frac{1}{q}} .
$$

For $m \in(1, \infty)$, let $D^{\alpha}\left(\mathbb{R}^{N}\right)$ denote the completion of $C_{0}^{\infty}\left(\mathbb{R}^{N}\right)$ with respect to the Gagliardo(semi) norm

$$
[u]_{\alpha}=\left(\int_{R^{N} R^{N}} \frac{|u(x)-u(y)|^{2}}{|x-y|^{N+2 \alpha}} d x d y\right)^{\frac{1}{2}}
$$

We define the space

$$
E_{\omega}=\left\{u \in D^{\alpha}\left(\mathbb{R}^{N}\right): \int_{R^{N}} \omega(x)|u(x)|^{2} d x<+\infty\right\}
$$

with the norm $\|\mathfrak{u}\|_{E_{\omega}}:=\left([u]_{\alpha}^{2}+\|u\|_{\omega}^{2}\right)^{1 / 2}$, where $\|\mathfrak{u}\|_{\omega}^{2}=\left(\int_{R^{N}} \omega(x)|u(x)|^{2} d x\right)^{1 / 2}$. It is easy to see the embedding $\mathrm{E}_{\omega} \longmapsto \mathrm{L}^{2}\left(\mathbb{R}^{\mathrm{N}}\right)$ is continuous. 
Denote

$$
X:=E_{V_{1}} \times E_{V_{2}}
$$

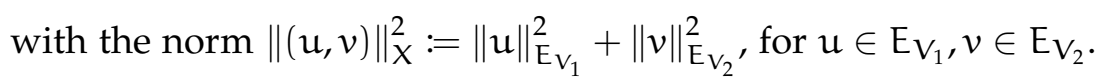

Similar to the discussion in $[27,36]$, we can get the following Lemmas 2.1-2.4.

Lemma 2.1. $X=\left(X,\|\cdot\|_{X}\right)$ is a separable and reflexive Banach space.

Lemma 2.2. The embedding $X \longmapsto L^{p}\left(\mathbb{R}^{N}\right) \times L^{p}\left(\mathbb{R}^{N}\right)$ is continuous, for $p \in\left[2,2_{\alpha}^{*}\right]$, and there exists a positive constant $\mathrm{C}_{\mathrm{p}}$ such that

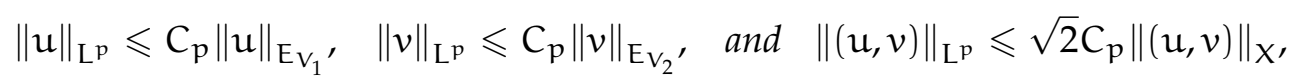

where $\|(\mathrm{u}, v)\|_{\mathrm{L}^{\mathrm{p}}\left(\mathbb{R}^{\mathrm{N}}\right)}=\left\|\sqrt{\mathrm{u}^{2}+v^{2}}\right\|_{\mathrm{L}^{\mathrm{p}\left(\mathbb{R}^{\mathrm{N}}\right)}}$.

Lemma 2.3. Let $\left\{\left(u_{n}, v_{n}\right)\right\}_{n} \subset X$ be such that $\left(u_{n}, v_{n}\right) \rightarrow(u, v)$ weakly in $X$ as $n \rightarrow \infty$. Then there exists a subsequence, which we also label it as $\left(u_{n}, v_{n}\right)$, such that $\left(u_{n}, v_{n}\right) \rightarrow(u, v)$ a.e. in $\mathbb{R}^{N}$ as $n \rightarrow \infty$.

Lemma 2.4. Assume the assumptions $\left(\mathrm{G}_{1}\right)-\left(\mathrm{G}_{2}\right)$ hold. Then the embeddings $\mathrm{E}_{\mathrm{V}_{1}} \longmapsto \mathrm{L}^{\mathrm{p}}\left(\mathbb{R}^{\mathrm{N}}\right)$ and $\mathrm{E}_{\mathrm{V}_{2}} \longmapsto$ $\mathrm{L}^{\mathrm{p}}\left(\mathbb{R}^{\mathrm{N}}\right)$ are compact. Moreover, the embedding $\mathrm{X} \longmapsto \mathrm{L}^{\mathrm{p}}\left(\mathbb{R}^{\mathrm{N}}\right) \times \mathrm{L}^{\mathrm{p}}\left(\mathbb{R}^{\mathrm{N}}\right)$ is compact for $\mathrm{p} \in\left[2,2_{\alpha}^{*}\right)$.

We consider the functionals $A, B, \varphi_{\lambda}: X \rightarrow R$, defined by

$$
\begin{aligned}
A(u, v)= & \frac{1}{2}\left[\int_{R^{N}} \int_{R^{N}} \frac{|u(x)-u(y)|^{2}}{|x-y|^{N+2 \alpha}} d x d y+\int_{R^{N}} v_{1}(x)|u(x)|^{2} d x\right] \\
& +\frac{1}{2}\left[\int_{R^{N}} \int_{R^{N}} \frac{|v(x)-v(y)|^{2}}{|x-y|^{N+2 \alpha}} d x d y+\int_{R^{N}} v_{2}(x)|v(x)|^{2} d x\right]=\frac{\|(u, v)\|_{X}^{2}}{2}, \\
B(u, v)= & \int_{R^{N}} F(x, u(x), v(x)) d x+\frac{1}{2_{\alpha}^{*}} \int_{R^{N}}\left(|u(x)|^{2_{\alpha}^{*}}+|v(x)|^{2_{\alpha}^{*}}\right) d x, \\
\varphi_{\lambda}(u, v)= & A(u, v)-\lambda B(u, v) \\
= & \frac{\|(u, v)\|_{X}^{2}}{2}-\lambda\left[\int_{R^{N}} F(x, u(x), v(x)) d x+\frac{1}{2_{\alpha}^{*}} \int_{R^{N}}\left(|u(x)|^{2_{\alpha}^{*}}+|v(x)|^{2_{\alpha}^{*}}\right) d x\right]
\end{aligned}
$$

for all $(u, v) \in X$ and $\lambda \in[1,2]$. Then $A, B, \varphi_{\lambda}$ is well-defined and continuously differentiable under the assumption $\left(\mathrm{H}_{0}\right)-\left(\mathrm{H}_{3}\right)$, and we have

$$
\begin{aligned}
\left\langle\varphi_{\lambda}^{\prime}(u, v),(t, z)\right\rangle= & \int_{R^{N}} \int_{R^{N}} \frac{[u(x)-u(y)][t(x)-t(y)]}{|x-y|^{N+2 \alpha}} d x d y+\int_{R^{N}} V_{1}(x) u t d x \\
& +\int_{R^{N}} \int_{R^{N}} \frac{[v(x)-v(y)][z(x)-z(y)]}{|x-y|^{N+2 \alpha}} d x d y+\int_{R^{N}} V_{2}(x) v z d x \\
& -\lambda\left[\int_{R^{N}}\left(F_{u}(x, u, v) t+F_{v}(x, u, v) z\right) d x+\int_{R^{N}}\left(|u(x)|^{2_{\alpha}^{*}-2} u t+|v(x)|^{2_{\alpha}^{*}-2} v z\right) d x\right]
\end{aligned}
$$

for all $(t, z) \in X$. Hence the critical point of $\varphi_{1}$ is the weak solution of problem (1.1). Next, we only consider the critical point of $\varphi_{1}$.

Let $\left\{e_{j}\right\}$ be a total orthonormal basis of $X$. We define

$$
X_{j}:=\operatorname{span}\left\{e_{j}\right\}, \quad Y_{k}:=\bigoplus_{j=1}^{k} X_{j}, \quad Z_{k}:=\overline{\bigoplus_{j=k+1}^{\infty} X_{j}}, \quad k \in \mathbb{N},
$$


and

$$
B_{k}=\left\{u \in Y_{k}:\|u\| \leqslant \rho_{k}\right\}, \quad S_{k}=\left\{u \in Z_{k}:\|u\|=r_{k}\right\}
$$

for $\rho_{k}>r_{k}>0$. Clearly, $X=Y_{k} \oplus Z_{k}$ with $\operatorname{dim} Y_{k}<\infty$.

The following variant fountain theorem will be used to prove the existence of infinitely many nontrivial small energy solutions of the system (1.1).

Lemma 2.5 ([39]). Let $\mathrm{X}$ be a Banach space, assume that $\varphi_{\lambda}(\mathrm{u})$ satisfies:

$\left(A_{1}\right) \varphi_{\lambda}(u)$ maps bounded sets into bounded sets uniformly for $\lambda \in[1,2]$, and

$$
\varphi_{\lambda}(-\mathfrak{u})=\varphi_{\lambda}(\mathfrak{u}), \quad \forall \lambda \in[1,2]
$$

$\left(\mathrm{A}_{2}\right) \mathrm{B}(\mathrm{u}) \geqslant 0$ for all $\mathrm{u} \in \mathrm{X}$, and $\mathrm{B}(\mathrm{u}) \rightarrow \infty$ as $\|\mathrm{u}\| \rightarrow \infty$ on any finite dimensional subspace of $\mathrm{X}$;

$\left(A_{3}\right)$ there exist $\rho_{k}>r_{k}>0$ such that

and

$$
a_{k}(\lambda):=\inf _{u \in Z_{k},\|u\|=\rho_{k}} \varphi_{\lambda}(u) \geqslant 0>b_{k}(\lambda):=\max _{u \in Y_{k},\|u\|=r_{k}} \varphi_{\lambda}(u), \quad \forall \lambda \in[1,2],
$$

$$
\mathfrak{c}_{k}(\lambda):=\inf _{\mathfrak{u} \in Z_{k},\|\mathfrak{u}\| \leqslant \rho_{k}} \varphi_{\lambda}(\mathfrak{u}) \rightarrow 0, \text { as } k \rightarrow \infty \text { uniformly for } \lambda \in[1,2] .
$$

Then there exist $\lambda_{\mathrm{n}} \rightarrow 1, \mathrm{u}\left(\lambda_{\mathrm{n}}\right) \in \mathrm{Y}_{\mathrm{n}}$ such that

$$
\varphi_{\lambda_{n}}^{\prime} \mid Y_{n}\left(u\left(\lambda_{n}\right)\right)=0 \text {, and } \varphi_{\lambda_{n}}\left(u\left(\lambda_{n}\right)\right) \rightarrow \omega_{k} \in\left[c_{k}(2), b_{k}(1)\right] \text {, as } n \rightarrow \infty \text {. }
$$

In particular, if $\left\{u\left(\lambda_{n}\right)\right\}$ has a convergent subsequence for every $\mathrm{k} \in \mathrm{N}$, then $\varphi_{1}$ has infinitely many nontrivial critical points $\left\{u_{k}\right\} \in X \backslash\{0\}$ satisfying $\varphi_{1}\left(u_{k}\right) \rightarrow 0^{-}$as $k \rightarrow \infty$.

\section{Main results}

Without loss of generality, we use the same notation $\left\{u_{n}\right\}$ for a sequence $\left\{u_{n}\right\}$ and any of its subsequence when it is needed.

Lemma 3.1. Assume $\left(\mathrm{G}_{1}\right)-\left(\mathrm{G}_{2}\right),\left(\mathrm{H}_{0}\right)-\left(\mathrm{H}_{3}\right)$ hold. Then, there exist $\rho_{\mathrm{k}}>\mathrm{r}_{\mathrm{k}}>0$ such that

and

$$
a_{k}(\lambda):=\inf _{(u, v) \in Z_{k},\|(u, v)\|_{x}=\rho_{k}} \varphi_{\lambda}(u) \geqslant 0>b_{k}(\lambda):=\max _{(u, v) \in Y_{k},\|(u, v)\|_{x}=r_{k}} \varphi_{\lambda}(u), \quad \forall \lambda \in[1,2],
$$

$$
c_{k}(\lambda):=\inf _{(u, v) \in Z_{k},\|(u, v)\|_{X} \leqslant \rho_{k}} \varphi_{\lambda}(u) \rightarrow 0, \text { as } k \rightarrow \infty \text { uniformly for } \lambda \in[1,2] .
$$

Proof. $\left(\mathrm{H}_{0}\right)-\left(\mathrm{H}_{3}\right)$ imply that for arbitrary $\delta>0$ with $\mathrm{C}_{2}^{2} \delta<\frac{1}{24}$, there exists a constant $\mathrm{d}_{\delta}$ such that

$$
\begin{aligned}
& F(x, u, v) \leqslant \delta|(u, v)|^{2}+d_{\delta} a|(u, v)|^{r}, \quad \forall(x, u, v) \in \mathbb{R}^{N} \times \mathbb{R}^{2}, \\
& F(x, u, v) \geqslant b|(u, v)|^{\sigma}-\delta|(u, v)|^{2}-d_{\delta} a|(u, v)|^{r}, \quad \forall(x, u, v) \in \mathbb{R}^{N} \times \mathbb{R}^{2},
\end{aligned}
$$

where $C_{2}$ was defined in Lemma 2.2.

From Lemma 2.2, (2.1), (3.1), for any $(u, v) \in Z_{k}, r \in[1,2), \lambda \in[1,2]$, we have

$$
\varphi_{\lambda}(u, v)=\frac{\|(u, v)\|_{X}^{2}}{2}-\lambda\left[\int_{R^{N}} F(x, u(x), v(x)) d x+\frac{1}{2_{\alpha}^{*}} \int_{R^{N}}\left(|u(x)|^{2_{\alpha}^{*}}+|v(x)|^{2_{\alpha}^{*}}\right) d x\right]
$$




$$
\begin{aligned}
& \geqslant \frac{\|(u, v)\|_{X}^{2}}{2}-\lambda\left[\delta 2 C_{2}^{2}\|(u, v)\|_{X}^{2}+d_{\delta} a 2^{\frac{r}{2}} C_{r}^{r}\|(u, v)\|_{X}^{r}\right]-\frac{\lambda}{2_{\alpha}^{*}} \int_{R^{N}}\left(|u(x)|^{2}+|v(x)|^{2}\right)^{\frac{2_{\alpha}^{*}}{2}} d x \\
& \geqslant \frac{\|(u, v)\|_{X}^{2}}{3}-d_{\delta} a 2^{1+\frac{r}{2}} C_{r}^{r}\|(u, v)\|_{X}^{r}-\frac{2}{2_{\alpha}^{*}}\|(u, v)\|_{L_{\alpha}^{2 *}}^{2_{\alpha}^{*}} .
\end{aligned}
$$

Let

$$
\alpha_{k}=\sup _{(u, v) \in Z_{k},\|(u, v)\|_{X}=1}\|(u, v)\|_{L^{p}}, \quad \text { for } p \in\left[2,2_{\alpha}^{*}\right) .
$$

By Lemma 2.5, similar to the argument of [34, Lemma 3.8], we can deduce that $\alpha_{k} \rightarrow 0$ as $k \rightarrow \infty$. Then, it follows from (3.3) that

$$
\varphi_{\lambda}(u) \geqslant \frac{\|(u, v)\|_{X}^{2}}{3}-d_{\delta} a 2^{1+\frac{r}{2}} C_{r}^{r}\|(u, v)\|_{X}^{r}-\frac{2}{2_{\alpha}^{*}} \alpha_{k}^{2_{\alpha}^{*}}\|(u, v)\|_{X}^{2_{\alpha}^{*}}
$$

Assume $\rho_{k}>\left(\frac{7}{2} d_{\delta} a 2^{1+\frac{r}{2}} C_{r}^{r}\right)^{\frac{1}{2-r}}$, then for any $\lambda \in[1,2], r \in[1,2)$, we have

$$
\begin{aligned}
a_{k}(\lambda) & :=\inf _{(u, v) \in Z_{k},\|(u, v)\|_{X}=\rho_{k}} \varphi_{\lambda}(u) \geqslant\|(u, v)\|_{X}^{r}\left[\frac{1}{3}\|(u, v)\|_{X}^{2-r}-d_{\delta} a 2^{1+\frac{r}{2}} C_{r}^{r}\right]-\frac{2}{2_{\alpha}^{*}} \alpha_{k}^{2_{\alpha}^{*}}\|(u, v)\|_{X}^{2_{\alpha}^{*}} \\
& \geqslant\left(\frac{7}{2} d_{\delta} a 2^{1+\frac{r}{2}} C_{r}^{r}\right)^{\frac{r}{2-r}}\left[\frac{d_{\delta} a 2^{1+\frac{r}{2}} C_{r}^{r}}{6}-\frac{2\|(u, v)\|_{X}^{2_{\alpha}^{*}-r}}{2_{\alpha}^{*}} \alpha_{k}^{2_{\alpha}^{*}}\right]>0, \quad \text { as } k \rightarrow \infty .
\end{aligned}
$$

Moreover, from (3.4), for any $(u, v) \in Z_{k},\|(u, v)\|_{X} \leqslant \rho_{k}, \lambda \in[1,2]$, one has

$$
\varphi_{\lambda}(u, v) \geqslant-\frac{2}{2_{\alpha}^{*}}\|(u, v)\|_{X}^{2_{\alpha}^{*}} \alpha_{k}^{2_{\alpha}^{*}} \rightarrow 0^{+}, \text {as } k \rightarrow \infty .
$$

Hence, from (2.1), for $(u, v) \in Z_{k},\|(u, v)\|_{X} \rightarrow 0$, and $\alpha_{k} \rightarrow 0^{+}$, as $k \rightarrow \infty$ it follows that

$$
c_{k}(\lambda):=\inf _{(u, v) \in Z_{k},\|(u, v)\|_{X} \leqslant \rho_{k}} \varphi_{\lambda}(u, v) \rightarrow 0, \text { as } k \rightarrow \infty \text { uniformly for } \lambda \in[1,2] \text {. }
$$

Next we will verify $b_{k}(\lambda):=\max _{(u, v) \in Y_{k},\|(u, v)\|_{X}=r_{k}} \varphi_{\lambda}(u, v)<0$, for all $\lambda \in[1,2]$. From (2.1), (3.2), for any $(u, v) \in Y_{k}$ with $\operatorname{dim} Y_{k}<\infty, \lambda \in[1,2]$, we have

$$
\begin{aligned}
\varphi_{\lambda}(u, v) & =\frac{\|(u, v)\|_{X}^{2}}{2}-\lambda\left[\int_{R^{N}} F(x, u(x), v(x)) d x+\frac{1}{2_{\alpha}^{*}} \int_{R^{N}}\left(|u(x)|^{2_{\alpha}^{*}}+|v(x)|^{2_{\alpha}^{*}}\right) d x\right], \\
& \leqslant \frac{\|(u, v)\|_{X}^{2}}{2}-\lambda \int_{R^{N}}\left(b|(u, v)|^{\sigma}-\delta|(u, v)|^{2}-d_{\delta} a|(u, v)|^{r}\right) d x .
\end{aligned}
$$

By the equivalence of any norm in finite dimensional space $Y_{k}$, it follows that

$$
\begin{aligned}
\varphi_{\lambda}(u, v) & \leqslant \frac{\|(u, v)\|_{X}^{2}}{2}+e_{1}\|(u, v)\|_{X}^{2}+e_{2}\|(u, v)\|_{X}^{r}-e_{3}\|(u, v)\|_{X}^{\sigma} \\
& =\|(u, v)\|_{X}^{\sigma}\left[\left(\frac{1}{2}+e_{1}\right)\|(u, v)\|_{X}^{2-\sigma}+e_{2}\|(u, v)\|_{X}^{r-\sigma}-e_{3}\right],
\end{aligned}
$$

where $e_{1}, e_{2}, e_{3}$ are positive constants. Then for $r \in[1,2)$, we can easily choose $0<\|(u, v)\|_{X}=r_{k}<\rho_{k}$ small enough such that

$$
b_{k}(\lambda):=\max _{(u, v) \in Y_{k},\|(u, v)\|_{X}=r_{k}} \varphi_{\lambda}(u, v), \quad \forall \lambda \in[1,2] .
$$

We complete the proof of Lemma 3.1. 
Lemma 3.2. Assume that $\left(\mathrm{G}_{1}\right)-\left(\mathrm{G}_{2}\right),\left(\mathrm{H}_{0}\right)-\left(\mathrm{H}_{3}\right)$ hold. Then, for $\lambda_{\mathrm{n}} \in[1,2], \lambda_{\mathrm{n}} \rightarrow 1$ and $\left(\mathrm{u}\left(\lambda_{\mathrm{n}}\right), v\left(\lambda_{\mathrm{n}}\right)\right) \in \mathrm{Y}_{\mathrm{n}}$ with

$$
\varphi_{\lambda_{n}}^{\prime} \mid \gamma_{n}\left(u\left(\lambda_{n}\right), v\left(\lambda_{n}\right)\right)=0 \text {, and } \varphi_{\lambda_{n}}\left(u\left(\lambda_{n}\right), v\left(\lambda_{n}\right)\right) \rightarrow \omega_{k} \in\left[c_{k}(2), b_{k}(1)\right] \text {, as } n \rightarrow \infty,
$$

$\left\{\left(u\left(\lambda_{n}\right), v\left(\lambda_{n}\right)\right)\right\}$ has a convergent subsequence in $X$ for every $k \in N$.

Proof. Assume that for each $k \in N, \lambda \in[1,2]$, there exist a subsequence $\lambda_{n} \rightarrow 1$, and $\left(u\left(\lambda_{n}\right), v\left(\lambda_{n}\right)\right) \in Y_{n}$ such that

$$
\varphi_{\lambda_{n}}^{\prime} \mid Y_{n}\left(u\left(\lambda_{n}\right), v\left(\lambda_{n}\right)\right)=0 \text {, and } \varphi_{\lambda_{n}}\left(u\left(\lambda_{n}\right), v\left(\lambda_{n}\right)\right) \rightarrow w_{k} \in\left[c_{k}(2), b_{k}(1)\right] \text {, as } n \rightarrow \infty .
$$

From (2.1), (3.1), $\left(H_{3}\right)$ and the condition $C_{2}^{2} \delta<\frac{1}{24}$, for $\lambda_{n} \in[1,2], \lambda_{n} \rightarrow 1$, as $n \rightarrow \infty$ and $r \in[1,2)$, one has

$$
\begin{aligned}
\frac{\|(u, v)\|_{X}^{2}}{2} & =\varphi_{\lambda}(u, v)+\lambda\left[\int_{R^{N}} F(x, u(x), v(x)) d x+\frac{1}{2_{\alpha}^{*}} \int_{R^{N}}\left(|u(x)|^{2_{\alpha}^{*}}+|v(x)|^{2_{\alpha}^{*}}\right) d x\right] \\
& \leqslant\left(\omega_{k}+1\right)+\lambda_{n}\left[\int_{R^{N}}\left(\delta|(u, v)|^{2}+d_{\delta} a|(u, v)|^{r}\right) d x+\frac{1}{2_{\alpha}^{*}} \int_{R^{N}}\left(|u(x)|^{2_{\alpha}^{*}}+|v(x)|^{2_{\alpha}^{*}}\right) d x\right] \\
& \leqslant\left(\omega_{k}+1\right)+\frac{\|(u, v)\|_{X}^{2}}{6}+2^{1+\frac{r}{2}} d_{\delta} a C_{r}^{r}\|(u, v)\|_{X}^{r}+\frac{2^{1+\frac{2_{\alpha}^{*}}{2}}}{2_{\alpha}^{*}} C_{2_{\alpha}^{*}}^{2^{*}}\|(u, v)\|_{X}^{2_{\alpha}^{*}},
\end{aligned}
$$

which shows that $\left\{\left(u\left(\lambda_{n}\right), v\left(\lambda_{n}\right)\right)\right\}$ is bounded in $X$. Then we can obtain a weakly convergent subsequence of $\left\{\left(u\left(\lambda_{n}\right), v\left(\lambda_{n}\right)\right)\right\}$. Assume that $\left\{\left(u\left(\lambda_{n}\right), v\left(\lambda_{n}\right)\right)\right\} \rightarrow(u, v)$ weakly in X. By Lemma 2.3, we know that $\left(u\left(\lambda_{n}\right), v\left(\lambda_{n}\right)\right) \rightarrow(u, v)$ strongly in $\mathbb{R}^{N}$, as $n \rightarrow \infty$. Then we have $\left|\left(u\left(\lambda_{n}\right)-u, v\left(\lambda_{n}\right)-v\right)\right| \rightarrow 0$ as $n \rightarrow \infty$, which implies

$$
\left|\mathfrak{u}\left(\lambda_{\mathrm{n}}\right)-\mathfrak{u}\right| \rightarrow 0 \text { and }\left|v\left(\lambda_{\mathrm{n}}\right)-v\right| \rightarrow 0, \text { as } \mathrm{n} \rightarrow \infty .
$$

Next we prove that $\left(u\left(\lambda_{n}\right), v\left(\lambda_{n}\right)\right) \rightarrow(u, v)$ in X. By (2.1)-(2.2), we have

$$
\begin{aligned}
\left\|\left(u\left(\lambda_{n}\right)-u, v\left(\lambda_{n}\right)-v\right)\right\|_{X}^{2} \leqslant & \left\langle\varphi_{\lambda_{n}}^{\prime}\left(u\left(\lambda_{n}\right), v\left(\lambda_{n}\right)\right)-\varphi_{1}^{\prime}(u, v),\left(u\left(\lambda_{n}\right)-u, v\left(\lambda_{n}\right)-v\right)\right\rangle \\
& +\int_{R^{N}}\left(\left|u\left(\lambda_{n}\right)-u\right|^{2 *}-2\left|u\left(\lambda_{n}\right)-u\right|^{2}+\left|v\left(\lambda_{n}\right)-v\right|^{2_{\alpha}^{*}-2}\left|v\left(\lambda_{n}\right)-v\right|^{2}\right) d x \\
& +\int_{R^{N}}\left[\lambda_{n} F_{u}\left(x, u\left(\lambda_{n}\right), v\right)-F_{u}(x, u, v)\right]\left(u\left(\lambda_{n}\right)-u\right) d x \\
& +\int_{R^{N}}\left[\lambda_{n} F_{v}\left(x, u, v\left(\lambda_{n}\right)\right)-F_{v}(x, u, v)\right]\left(v\left(\lambda_{n}\right)-v\right) d x .
\end{aligned}
$$

It is easy to see that

$$
\left\langle\varphi_{\lambda_{n}}^{\prime}\left(u\left(\lambda_{n}\right), v\left(\lambda_{n}\right)\right)-\varphi_{1}^{\prime}(u, v),\left(u\left(\lambda_{n}\right)-u, v\left(\lambda_{n}\right)-v\right)\right\rangle, \quad n \rightarrow \infty .
$$

From (3.5), one has

$$
\int_{R^{N}}\left(\left|u\left(\lambda_{n}\right)-u\right|^{2_{\alpha}^{*}-2}\left|u\left(\lambda_{n}\right)-u\right|^{2}+\left|v\left(\lambda_{n}\right)-v\right|^{2_{\alpha}^{*}-2}\left|v\left(\lambda_{n}\right)-v\right|^{2}\right) d x \rightarrow 0, \quad n \rightarrow \infty .
$$

From $\left(\mathrm{H}_{1}\right), \lambda_{m} \rightarrow 1,\left\{\left(u\left(\lambda_{n}\right), v\left(\lambda_{n}\right)\right)\right\}$ is bounded in $X$, and $\left(u\left(\lambda_{n}\right), v\left(\lambda_{n}\right)\right) \rightarrow(u, v)$ strongly in $\mathbb{R}^{N}$, we 
have

$$
\begin{aligned}
\int_{R^{N}}\left[\lambda_{n} F_{u}\left(x, u\left(\lambda_{n}\right), v\right)-\right. & \left.F_{u}(x, u, v)\right]\left(u\left(\lambda_{n}\right)-u\right) d x+\int_{R^{N}}\left[\lambda_{n} F_{v}\left(x, u, v\left(\lambda_{n}\right)\right)-F_{v}(x, u, v)\right]\left(v\left(\lambda_{n}\right)-v\right) d x \\
\leqslant & \int_{R^{N}}\left[\left|2 F_{u}\left(x, u\left(\lambda_{n}\right), v\right)\right|+\left|F_{u}(x, u, v) \| u\left(\lambda_{n}\right)-u\right|\right] d x \\
& +\int_{R^{N}}\left[\left|2 F_{v}\left(x, u, v\left(\lambda_{n}\right)\right)\right|+\left|F_{v}(x, u, v)\right|\left|v\left(\lambda_{n}\right)-v\right|\right] d x \\
\leqslant & 3 a\left(1+|z|^{r-1}\right)\left[\left|u\left(\lambda_{n}\right)-u\right|+\left|v\left(\lambda_{n}\right)-v\right|\right] \rightarrow 0, \quad n \rightarrow \infty .
\end{aligned}
$$

Equations (3.6), (3.7), (3.8), (3.9) show $\left\|\left(u\left(\lambda_{n}\right)-u, v\left(\lambda_{n}\right)-v\right)\right\|_{X}^{2} \rightarrow 0$, which implies that $\left(u\left(\lambda_{n}\right), v\left(\lambda_{n}\right)\right) \rightarrow$ $(u, v)$ in $X$. The proof is completed.

Proof of Theorem 1.1. Obviously, from $\left(\mathrm{H}_{0}\right)$, we know $B(u) \geqslant 0$ and

$$
\mathrm{B}(\mathrm{u}, v)=\int_{\mathrm{R}^{\mathrm{N}}} \mathrm{F}\left(\mathrm{x}, \mathrm{u}(\mathrm{x}), v(\mathrm{x}) \mathrm{d} \mathrm{d} x+\frac{1}{2_{\alpha}^{*}} \int_{\mathrm{R}^{\mathrm{N}}}\left(|\mathrm{u}(\mathrm{x})|^{2_{\alpha}^{*}}+|v(x)|^{2_{\alpha}^{*}}\right) \mathrm{d} x \rightarrow \infty, \quad \text { as }\|(\mathrm{u}, v)\|_{X} \rightarrow \infty,\right.
$$

on any finite dimensional subspace of $X$, which implies $\left(A_{2}\right)$ of Lemma 2.5 is satisfied

From $\left(H_{0}\right),(2.1)$ and the condition $F(x,-u,-v)=F(x, u, v)$, we can verify $\varphi_{\lambda}(u, v)$ maps bounded sets into bounded sets uniformly for $\lambda \in[1,2]$, and

$$
\varphi_{\lambda}(-\mathfrak{u},-v)=\varphi_{\lambda}(u, v), \quad \forall(\lambda, u) \in[1,2] \times X,
$$

which shows $\left(A_{1}\right)$ of Lemma 2.5 holds. Lemma 3.1 implies that $\left(A_{3}\right)$ is also satisfied. In view of Lemma 2.5 , we know that for each $k \in N$, there exist $\lambda_{n} \rightarrow 1,\left(u\left(\lambda_{n}\right), v\left(\lambda_{n}\right)\right) \in Y_{n}$ such that

$$
\left.\varphi_{\lambda_{n}}^{\prime}\right|_{Y_{n}}\left(u\left(\lambda_{n}\right), v\left(\lambda_{n}\right)\right)=0 \text {, and } \varphi_{\lambda_{n}}\left(u\left(\lambda_{n}\right), v\left(\lambda_{n}\right)\right) \rightarrow \omega_{k} \in\left[c_{k}(2), b_{k}(1)\right] \text {, as } n \rightarrow \infty \text {. }
$$

By Lemma 3.2, we know $\left\{\left(u\left(\lambda_{n}\right), v\left(\lambda_{n}\right)\right)\right\}$ has a convergent subsequence in X. Owing to Lemma 2.5, $\varphi_{1}(u, v)$ has infinitely many nontrivial critical points $\left(u^{(\mathrm{k})}, v^{(\mathrm{k})}\right) \in X \backslash\{0\}$ satisfying that

$$
\frac{\left\|\left(u^{(k)}, v^{(k)}\right)\right\|_{X}^{2}}{2}-\left[\int_{R^{N}} F\left(x, u^{(k)}, v^{(k)}\right) d x+\frac{1}{2_{\alpha}^{*}} \int_{R^{N}}\left(\left|u^{(k)}\right|^{2_{\alpha}^{*}}+\left|v^{(k)}\right|^{2_{\alpha}^{*}}\right) d x\right] \rightarrow 0^{-}, \text {as } k \rightarrow \infty
$$

for every $k \in N$, which implies (1.1) possesses infinitely many small energy solutions.

Remark 3.3. If the sublinear case for the nonlinearity $F_{z}$ turns to the following superlinear case:

$\left(H_{1}^{\prime}\right)$ There exist two constants $a_{0}>0, v \in\left[2,2_{\alpha}^{*}\right)$ such that

$$
\left|\mathrm{F}_{z}(x, z)\right| \leqslant \mathrm{a}_{0}\left(1+|z|^{\nu-1}\right), \quad \forall(x, z) \in \mathbb{R}^{\mathrm{N}} \times \mathbb{R}^{2}, \quad z=(\mathrm{u}, v),
$$

where $F_{z}(x, z)=\left(F_{u}(x, z), F_{v}(x, z)\right),|z|=|(u, v)|=\sqrt{u^{2}+v^{2}}$.

$\left(\mathrm{H}_{2}\right) \lim _{|(u, v)| \rightarrow 0} \frac{\mathrm{F}(\mathrm{t}, \mathrm{u}, v)}{|(u, v)|^{2}}=0$ uniformly in $x \in \mathbb{R}^{\mathrm{N}}$.

$\left(H_{3}^{\prime}\right) \lim _{|(u, v)| \rightarrow \infty} \frac{F(t, u, v)}{|(u, v)|^{2}}=\infty$ uniformly in $t \in[0, T]$.

By using another variant fountain theorem in [39], we can also establish the existence criteria of infinitely many high energy solutions of the problem (1.1). 
Remark 3.4. As we know, the coupled quasilinear Schrödinger system appears in several branches of physics. For example, it can be used as a model for propagation of polarized laser beams in birefringent Kerr medium in nonlinear optics. Physically, the two functions $u$ and $v$ denote the components of the slowly varying envelope of the electrical field, $x$ are the orthogonal variables and $(-\Delta)^{\alpha}$ is the diffraction operator. The focusing nonlinear terms in (1.1) describe the dependence of the refraction index of the material on the electric field intensity and the birefringence effects. The existence of nonstandard small energy solutions of (1.1), that is, solutions with minimal energy on the set of solutions $(u, v)$ to the system such that $u \neq \equiv, v \not \equiv 0$, implies that the speed of the components of the varying envelope in the electrical field may be very slowly. Hence, the obtained results can describe the specific condition of the propagation of polarized laser beams in birefringent Kerr medium very clearly. In the other branches of physics, our main results represent other physical meaning.

\section{Conclusion}

In this paper, we study the existence of infinitely many small energy solutions for a coupled fractional Schrödinger system (1.1) with critical growth, which can describe the multiplicate chemical reaction catalyzed by the catalyst grains under constant or variant temperature, and a correspondence of the stable station of the dynamical system determined by the reactiondiffusion system very well. It should be noted that the coupled fractional Schrdinger system which we discuss is with critical growth and fractional order Laplacian. By the variational methods, the existence criteria of infinitely many small energy solutions for the problem (1.1) are established without A-R condition. Recent results in the literature are generalized and improved.

\section{Acknowledgment}

The authors thank the referees for their careful reading of the manuscript and insightful comments, which help to improve the quality of the paper. We would also like to acknowledge the valuable comments and suggestions from the editors, which vastly contribute to the perfection of the paper.

This work is supported by National Natural Science Foundation of China (No. 11301153, 61673008), China Postdoctoral Science Foundation (No. 2017M612392).

\section{References}

[1] W. M. Abd-Elhameed, Y. H. Youssri, Spectral solutions for fractional differential equations via a novel Lucas operational matrix of fractional derivatives, Rom. J. Phys., 61 (2016), 795-813. 1

[2] M. A. Abdelkawy, M. A. Zaky, A. H. Bhrawy, D. Baleanu, Numerical simulation of time variable fractional order mobile-immobile advection-dispersion model, Rom. Rep. Phys., 67 (2015), 1-19.

[3] A. Agila, D. Baleanu, R. Eid, B. Irfanoglu, Applications of the extended fractional Euler-Lagrange equations model to freely oscillating dynamical systems, Rom. J. Phys., 61 (2016), 350-359. 1

[4] P. Álvarez-Caudevilla, E. Colorado, V. A. Galaktionov, Existence of solutions for a system of coupled nonlinear stationary bi-harmonic Schrödinger equations, Nonlinear Anal. Real World Appl., 23 (2015), 78-93. 1, 1

[5] D. Applebaum, Léry processes-from probalility to fnance and quantum groups, Notices Amer. Math. Soc., 51 (2004), 1336-1347. 1

[6] D. Baleanu, S. I. Muslih, K. Taş, Fractional Hamiltonian analysis of higher order derivatives systems, J. Math. Phys., 2006 (2006), 8 pages. 1

[7] T. Bartsch, Z. Q. Wang, Existence and multiplicity results for some superlinear elliptic problems on $\mathbb{R}^{\mathrm{N}}$, Comm. Partial Differential Equations, 20 (1995), 1725-1741. 1

[8] A. H. Bhrawy, A new spectral algorithm for time-space fractional partial differential equations with subdiffusion and superdiffusion, Proc. Rom. Acad. Ser. A Math. Phys. Tech. Sci. Inf. Sci., 17 (2016), 39-47. 1

[9] G. M. Bisci, V. D. Rădulescu, Ground state solutions of scalar field fractional for Schrödinger equations, Calc. Var. Partial Differ. Equ., 54 (2015), 2985-3008. 1

[10] C. Chen, S. Fu, Infinitely many solutions to quasi-linear Schrödinger system in $\mathbb{R}^{N}$, Comput. Math. Appl., 71 (2016), 1417-1424. 1, 1

[11] D. G. Costa, On a class of elliptic systems in $\mathbb{R}^{N}$, Electron. J. Differential Equations, 1994 (1994), 14 pages. 1, 1 
[12] E. Di Nezza, G. Palatucci, E. Valdinoci, Hitchhikers guide to the fractional sobolev spaces, Bull. Sci. Math., 136 (2012), 521-573. 1

[13] P. Felmer, A. Quaas, J. Tan, Positive solutions of the nonlinear Schrödinger equation with the fractional Laplacian, Proc. R. Soc. Edinb., 142 (2012), 1237-1262. 1

[14] M. F. Furtado, E. A. B. Silva, M. S. Xavier, Multiplicity and concentration of solutions for elliptic systems with vanishing potentials, J. Differential Equations, 249 (2010), 2377-2396. 1, 1

[15] B. Ge, Multiple solutions of nonlinear Schrödinger equation with the fractional Laplacian, Nonlinear Anal. Real World Appl., 30 (2016), 236-247. 1

[16] Q. Guo, X. He, Least energy solutions for a weakly coupled fractional Schrödinger system, Nonlinear Anal., 132 (2016), 141-159. 1, 1

[17] A. A. Kilbas, H. M. Srivastava, J. J. Trujillo, Theory and Applications of Fractional Differential Equations, Elsevier Science, Amsterdam, (2006). 1

[18] D. Kumar, J. Singh, D. Baleanu, fractional model of convective radial fins with temperature-dependent thermal conductivity, Rom. Rep. Phys., 2017 (2017), 13 pages. 1

[19] V. Lakshmikantham, S. Leela, J. V. Devi, Theory of fractional dynamic systems, Cambridge Scientific Publishers, Cambridge, (2009). 1

[20] N. Laskin, Fractional quantum mechanics and Lévy path integrals, Phys. Lett. A, 268 (2000), 298-305. 1

[21] N. Laskin, Fractional Schrödinger equation, Phys. Rev. E, 2002 (2002), 7 pages. 1

[22] J.-Q. Liu, X.-Q. Liu, Z.-Q, Wang, Multiple mixed states of nodal solutions for nonlinear Schrdinger systems, Calc. Var. Partial Differential Equations, 52 (2015), 565-586. 1, 1

[23] D.-F. Lü, Q. Liu, Multiplicity of solutions for a class of quasilinear Schrödinger systems in $\mathbb{R}^{\mathrm{N}}$, Comput. Math. Appl., 66 (2014), 2532-2544. 1, 1

[24] S. I. Muslih, O. P. Agrawal, D. Baleanu, A Fractional Schrödinger Equation and Its Solution, Int. J. Theoretical Phys., 49 (2010), 1746-1752. 1

[25] K. Nouri, S. Elahi-Mehr, L. Torkzadeh, Investigation of the behavior of the fractional Bagley-Torvik and Basset equations via numerical inverse Laplace transform, Rom. Rep. Phys., 68 (2016), 503-514. 1

[26] I. Podlubny, Fractional differential equations, Academic Press, New York, (1999). 1

[27] P. Pucci, M. Q. Xiang, B. L. Zhang, Multiple solutions for nonhomogeneous SchrödingerKirchhoff type equations involving the fractional p-Laplacian in $\mathbb{R}^{\mathrm{N}}$, Calc. Var. Partial Differ. Equ., 54 (2015), 2785-2806. 1, 2

[28] U. Severo, E. Silva, On the existence of standing wave solutions for a class of quasilinear Schrödinger systems, J. Math. Anal. Appl., 412 (2014), 763-775. 1, 1

[29] X.-D. Shang, J.-H. Zhang, Ground states for fractional Schrödinger equations with critical growth, Nonlinearity, 27 (2014), 187-207. 1

[30] F. Tao, X. Wu, Existence and multiplicity of positive solutions for fractional Schrödinger equations with critical growth, Nonlinear Anal. Real World Appl., 35 (2017), 158-174. 1

[31] V. E. Tarasov, Fractional dynamics: application of fractional calculus to dynamics of particles, fields and media, Springer, New York, (2011). 1

[32] K. Teng, Multiple solutions for a class of fractional Schrödinger equations in $\mathbb{R}^{\mathrm{N}}$, Nonlinear Anal. Real World Appl., 21 (2015), 76-86. 1

[33] K. Teng, X. He, Ground state solutions for fractional Schrödinger equations with critical Sobolev exponent, Commun. Pure Appl. Anal., 15 (2016), 991-1008. 1

[34] M. Willem, Minimax Theorems, Birkhäuser, Boston, (1996). 3

[35] X.-J. Yang, F. Gao, H. M. Srivastava, New rheological models within local fractional derivative, Rom. Rep. Phys., 2017 (2017), 12 pages. 1

[36] L. Yang, Z. Liu, Multiplicity and concentration of solutions for fractional Schrödinger equation with sublinear perturbation and steep potential well, Comput. Math. Appl., 72 (2016), 1629-1640. 1, 2

[37] Y. Zhang, D. Baleanu, X.-J. Yang, New solutions of the transport equations in porous media within local fractional derivative, Proc. Rom. Acad. Ser. A Math. Phys. Tech. Sci. Inf. Sci., 17 (2016), 230-236. 1

[38] X. Zhang, B. Zhang, D. Repovš, Existence and symmetry of solutions for critical fractional Schrdinger equations with bounded potentials, Nonlinear Anal., 142 (2016), 48-68. 1

[39] W. Zou, Variant fountain theorems and their applications, Manuscripta Math., 104 (2001), 343-358. 2.5, 3.3 